\title{
Le LiDAR : un outil pour l'étude archéologique des usages anciens des sols
}

\author{
LiDAR as a tool to study past land-use
}

\section{Catherine Fruchart ${ }^{1}$}

${ }^{1}$ chercheuse associée à l'USR 3124 (MSHE, Besançon) et ingénieure de recherche contractuelle de l'USR 3124 (MSH, Dijon), UBFC, meyer.thy@orange.fr

Avec la collaboration de :

François Favory, UMR 6249 Chrono-Environnement CNRS - UBFC, professeur émérite d'histoire ancienne et archéologie gallo-romaine, favory.francois2@wanadoo.fr

RÉSUMÉ. Les vestiges de paysages passés nous sont parvenus et leur étude peut nous renseigner sur l'occupation ancienne des territoires. Le LiDAR, couplé aux sources de données traditionnelles, aux SIG et à la modélisation, ouvre de nouvelles perspectives pour mieux connaître les paysages passés et leurs transformations. Ces données montrent notamment la fréquence des vestiges parcellaires dans les forêts actuelles en France, occupant jusqu'à plusieurs centaines de kilomètres carrés d'un seul tenant. Ces vestiges matérialisent des espaces autrefois dédiés à des activités agro-sylvo-pastorales. 17 zones (environ $2200 \mathrm{~km} 2$ ), comportant des vestiges parcellaires attribués à l'Antiquité, ont été étudiées dans le cadre du programme ERC Rurland consacré aux dynamiques spatiales et historiques des territoires ruraux entre le bassin de la Seine et le limes de Germanie, de la Tène finale à l'Antiquité tardive. La moitié de ces zones, essentiellement sous forêt, intègre du LiDAR. Documentées par des données archéologiques, elles ont été mises en perspective avec des caractéristiques environnementales : pentes, textures de sol, capacités de réserve en eau du sol, degrés de fertilité ou productivité sylvicole, limites à usage agricole, et autres informations topographiques, géologiques et pédologiques. En outre, une étude détaillée des morphologies parcellaires a été opérée sur six zones pour mieux comprendre comment les parcellaires s'intègrent dans leur environnement. L'examen à différentes échelles de systèmes parcellaires a permis d'identifier quelques facteurs qui semblent favorables ou défavorables à un usage agro-pastoral passé. Une capacité de réserve en eau du sol insuffisante et une fertilité naturelle faible pourraient être des facteurs limitant cet usage dans l'Antiquité ; la présence de pentes a peut-être favorisé la déprise ancienne d'un usage agro-pastoral, après la période romaine.

ABSTRACT. The vestiges of past landscapes have come down to us and their study can inform us about the ancient occupation of the territories. LiDAR, coupled with traditional data sources, GIS and modelling, opens up new perspectives to better understand past landscapes and their transformations. In particular, these data show the frequency of patchy remains in present-day forests in France, occupying up to several hundred square kilometres in a single block. These vestiges materialize spaces formerly dedicated to agro-sylvo-pastoral activities. 17 areas (about $2200 \mathrm{~km} 2$ ), including parcel vestiges attributed to Antiquity, have been studied within the framework of the ERC Rurland programme devoted to the spatial and historical dynamics of rural territories between the Seine basin and the Limes de Germanie, from the Final Tène to Late Antiquity. Half of these areas, mainly under forest, incorporate LiDAR. Documented by archaeological data, they have been put into perspective with environmental characteristics: slopes, soil textures, soil water reserve capacities, degrees of fertility or silvicultural productivity, limits to agricultural use, and other topographical, geological and pedological information. In addition, a detailed study of plot morphologies was carried out in six areas to better understand how the plots fit into their environment. Examination at different scales of plot systems identified some factors that appear to favour or hinder past agro-pastoral use. Insufficient soil water reserve capacity and low natural fertility could be factors limiting this use in antiquity; the presence of slopes may have favoured the ancient abandonment of an agro-pastoral use after the Roman period.

MOTS-CLÉS. LiDAR, forêt, agro-pastoralisme, système parcellaire, usage du sol.

KEYWORDS. forest, agropastoralism, field system, land use.

\section{Introduction}

Ce que nous savons du cadre de vie des sociétés anciennes s'appuie surtout sur la connaissance archéologique des espaces bâtis et funéraires ; des données paléoenvironnementales, textes et sources graphiques complétant l'information. L'emprise spatiale des fenêtres d'étude est restreinte et, malgré 
les progrès techniques et conceptuels des dernières décennies en archéologie, la diversité et l'extension des territoires passés sont mal connues. Or, des vestiges des paysages passés nous sont parvenus. Leur étude peut nous renseigner sur les modalités anciennes d'occupation des territoires. Le LiDAR offre une excellente opportunité de développements dans ce domaine en révélant, par exemple, les vestiges de systèmes agraires, de réseaux viaires ou d'exploitation de ressources naturelles.

L'examen de levés LiDAR et les publications associées montrent la fréquence des vestiges parcellaires dans les forêts actuelles en France. Depuis le milieu des années 2000, des centaines de kilomètres carrés d'anciens systèmes parcellaires ont ainsi été détectés dans des forêts actuelles grâce à cette technique ${ }^{1}$. Un «système parcellaire » se définit comme un groupement de parcelles formant un ensemble cohérent, qui se reconnaît par l'identification de liens morphologiques associant les parcelles entre elles. Leurs morphologies et les contextes dans lesquels on les rencontre sont variés. Ils sont souvent difficiles à dater, la chronologie dépendant pour l'essentiel des contextes archéologiques (habitats, voies datées, etc.) pouvant leur être associés. Ces systèmes matérialisent l'espace autrefois dédié à des activités agro-sylvo-pastorales : anciens champs cultivés, prairies, pâtures, vignes, vergers, bois-taillis, etc. (Favory et Fruchart 2018). Leur identification vient enrichir des connaissances acquises depuis plusieurs décennies par l'étude de plans anciens, de photographies aériennes et parfois par des fouilles ${ }^{2}$.

\section{Systèmes parcellaires étudiés pour le programme Rurland}

Plusieurs systèmes parcellaires ont été étudiés dans le cadre du programme ERC Rurland ${ }^{3}$, consacré aux dynamiques spatiales et historiques des territoires ruraux entre le bassin de la Seine et le limes de Germanie, de la Tène finale à l'Antiquité tardive (Reddé 2017, 2018). Le programme Rurland a exploité des données de fouilles et de prospections, des données faunistiques, floristiques, géologiques et pédologiques, des photographies aériennes et du LiDAR. Les recherches sur les systèmes parcellaires (Favory et al. 2017 ; Favory et Fruchart 2018) ont concerné 17 zones, pour lesquelles les publications et données brutes disponibles permettaient une approche synthétique et comparative sur les formes d'occupation et d'usage des sols durant l'Antiquité. Les zones étudiées totalisent une surface d'environ $2200 \mathrm{~km}^{2}$ (fig. 1) dont la moitié, majoritairement sous forêt aujourd'hui, intègre du LiDAR (8 zones, $\left.1100 \mathrm{~km}^{2}\right)$.

\footnotetext{
${ }^{1}$ De façon non exhaustive, nous pouvons par exemple citer en France : Georges-Leroy et al. 2009, Étienne 2011, Laplaige 2012, Fruchart 2014, Froehlicher 2016, Le Jeune et Dardignac 2017, Favory et al. 2017, Crozet et al. 2018, Favory et Fruchart 2018, Goguey, Bénard 2018, Laüt 2018. En Allemagne, il y a notamment les travaux pionniers de Benoît Sittler (Heinzel et Sittler 2010) et plusieurs exemples au Royaume-Uni (Crow et al. 2007, Crutchley et Winton 2010, entre autres). En Slovénie et en Italie, des systèmes parcellaires en milieu alpin ont été étudiés (Stular 2011, Forlin 2012); aux Pays-Bas, ce sont des " Celtic » fields protohistoriques (Arnoldussen et Van der Linden 2017), en Mésoamérique, des aménagements précolombiens (Chase 2017), sur la côte est des USA, à Hawaï (McCoy et al. 2011) ou aux Caraïbes (Opitz et al. 2015), des parcellaires modernes (Johnson et Ouimet 2016), etc.

${ }^{2}$ Pour des références sur ce sujet, se reporter par exemple à la bibliographie de Favory et Fruchart 2018 ou aux références listées sur https://www.archeogeographie.org/index.php?rub=bibli/ouvrages (lien valide le 1111 2019).
}

${ }^{3}$ https://rurland.hypotheses.org/ (lien valide le 1111 2019). 


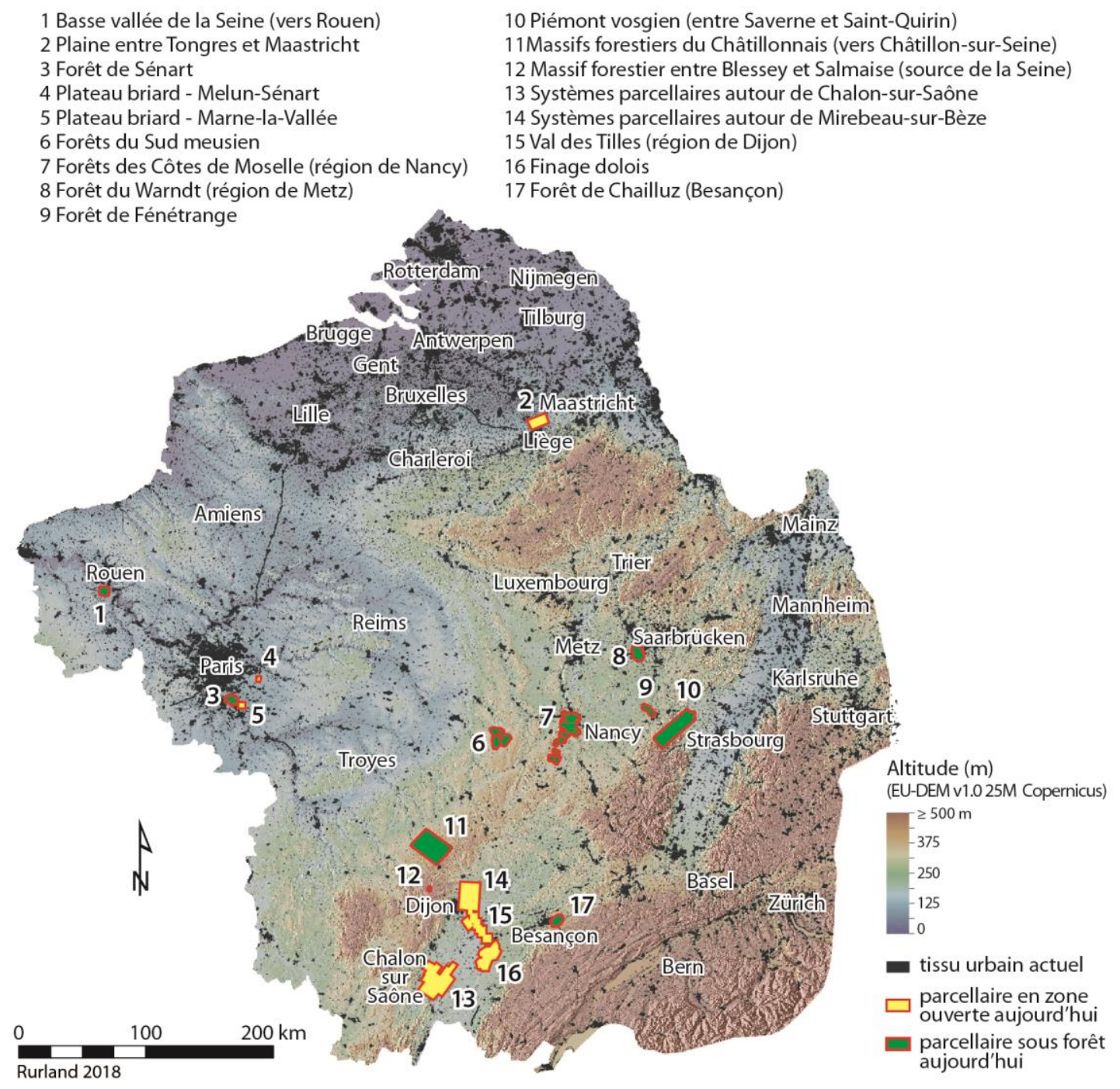

Fig. 1. Localisation des réseaux parcellaires étudiés dans l'aire géographique du programme ERC Rurland. C. Fruchart, EPHE 2018 et UBFC 2019, d'après Copernicus Land Monitoring Service, http://www.copernicus.eu/, EU-DEM v1.0.

Afin d'avoir, dans un premier temps, un point de vue global des caractéristiques a priori favorables ou défavorables à un usage agro-pastoral (passé ou actuel) des zones étudiées, les 17 fenêtres d'étude ont été mises en perspective avec des données environnementales extraites des bases européennes Copernicus et Soil Geographical Database of Eurasia (SGDBE).

Copernicus fournit un modèle numérique de terrain à moyenne échelle d'une résolution de 25 mètres, à partir duquel nous avons réalisé un modèle de pentes (fig. 2). En croisant l'occupation du sol actuelle ${ }^{4}$ avec la déclivité (fig. 3), nous constatons que les sols plats (pente inférieure à $2^{\circ}$ ) sont un peu plus fréquents pour les zones avec parcellaire (52\%) que pour la zone d'étude Rurland $(44 \%)^{5}$. Par ailleurs, les zones avec parcellaires attribués à l'Antiquité et sous forêt aujourd'hui présentent un taux de sols en pente (plus de $5^{\circ}$ ) beaucoup plus élevé $(40 \%)$ que celles qui sont en milieu ouvert aujourd'hui $(4 \%)$. Le caractère pentu pourrait avoir favorisé la déprise ancienne d'un usage agro-

\footnotetext{
${ }^{4}$ CORINE Land Cover 2012

${ }^{5}$ Les 17 zones étudiées occupent une surface trop limitée pour constituer un échantillon statistique représentatif de la zone d'étude Rurland. D'un point de vue statistique, nous pouvons toutefois comparer les distributions à l'intérieur des zones étudiées.
} 
pastoral, intervenue à une période indéterminée antérieure au $\mathrm{XIX}^{\mathrm{e}}$ siècle $^{6}$, pour les fenêtres étudiées. Une évolution similaire tardive, postérieure à 1860, a été identifiée dans le Parc naturel régional du Luberon, avec le boisement de terrains pentus autrefois dédiés à un usage agro-pastoral (Abadie et al. 2017).

\section{Systèmes parcellaires : zones étudiées et classes de pente}

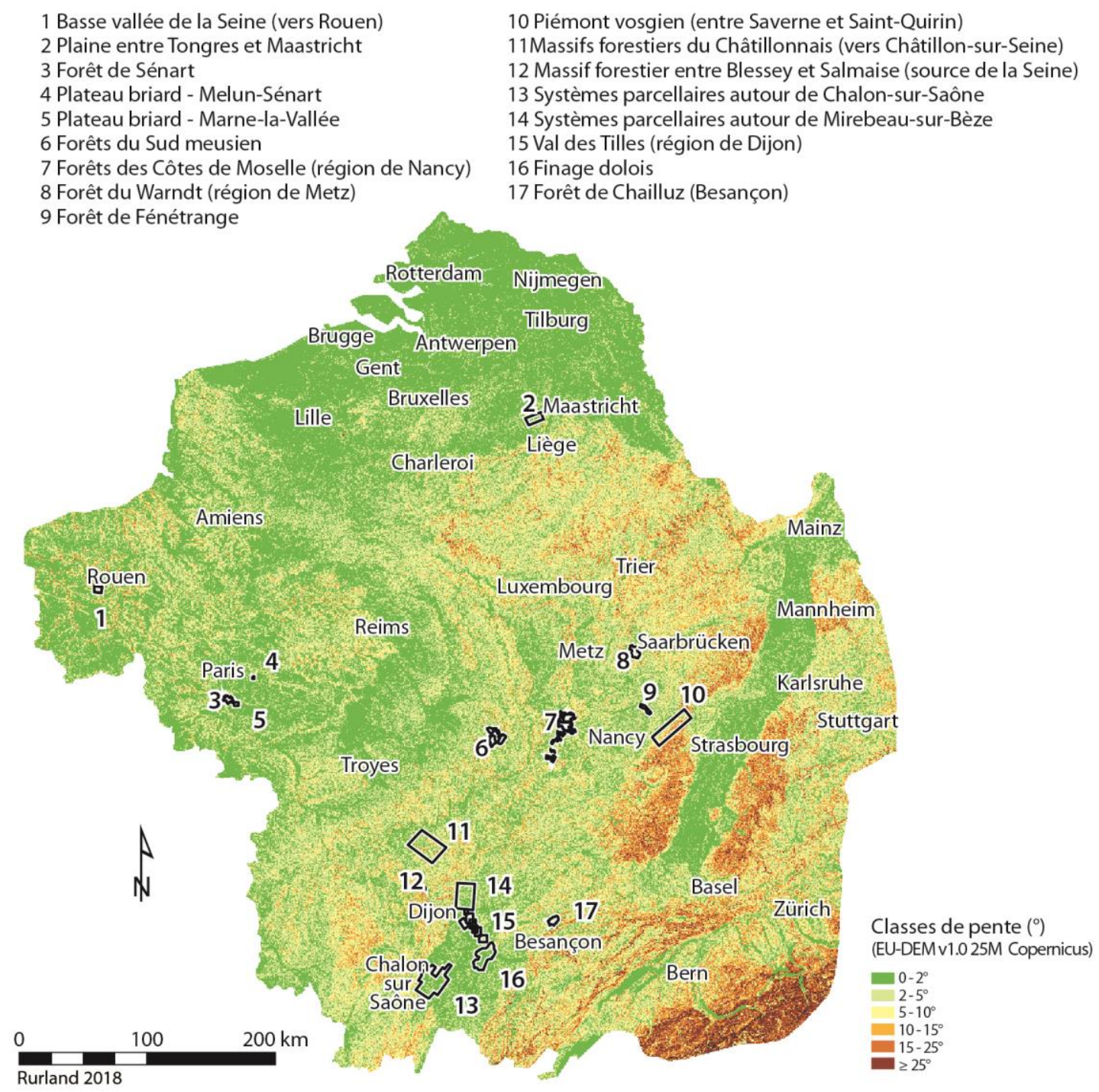

Fig. 2. Localisation des réseaux parcellaires étudiés sur un modèle de pentes. C. Fruchart, EPHE 2018 et UBFC 2019, d'après Copernicus Land Monitoring Service, http://www.copernicus.eu/, EU-DEM v1.0. Modèle de pentes C. Fruchart, 2018.

\footnotetext{
${ }^{6}$ Pour les zones étudiées, l'état boisé antérieur au XIX ${ }^{\mathrm{e}}$ siècle est attesté par des archives textuelles et/ou des plans et cartes des XVIII ${ }^{\mathrm{e}}$-XIX ${ }^{\mathrm{e}}$ siècles.
} 


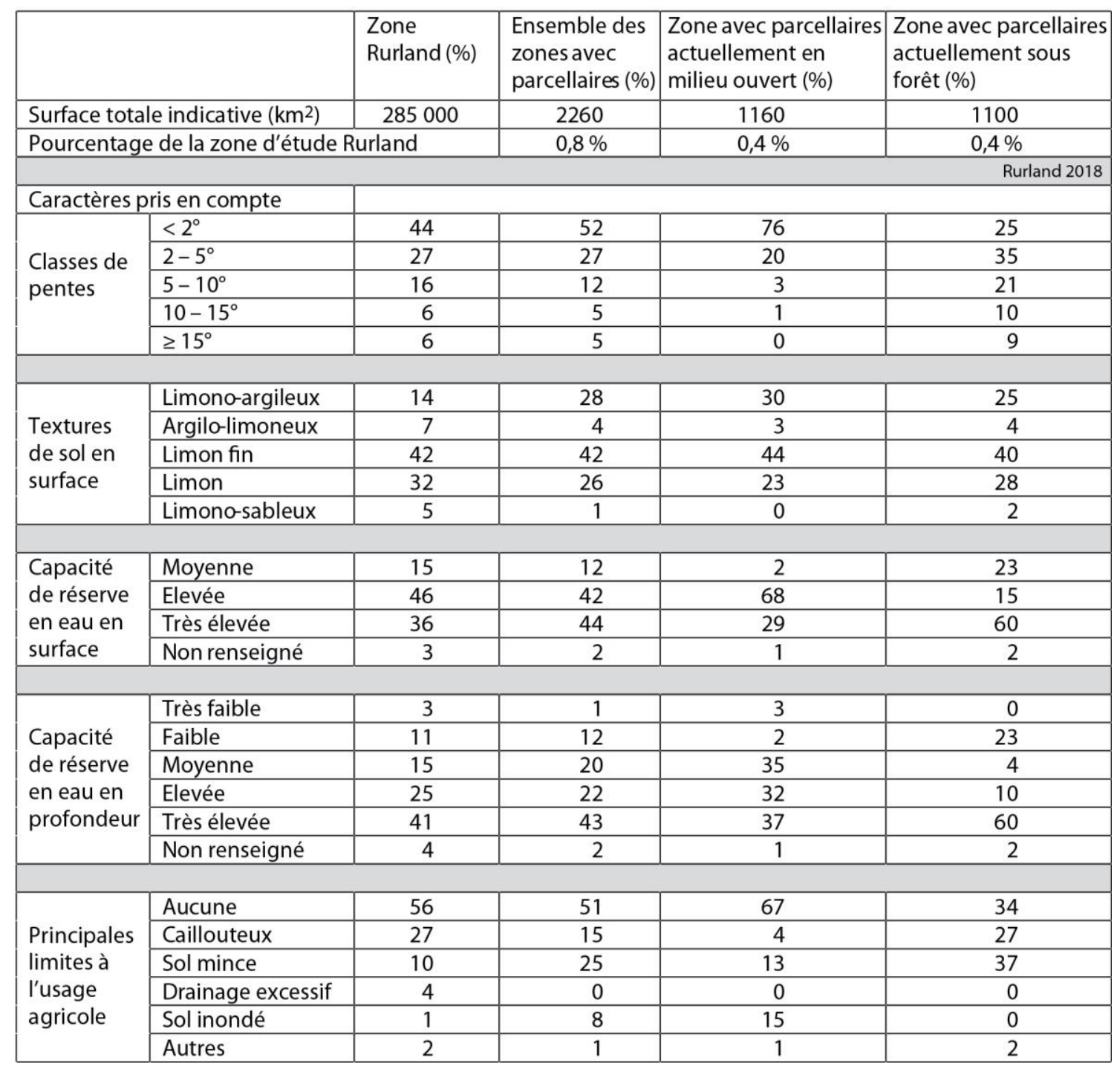

Fig. 3. Comparaison entre différentes caractéristiques environnementales de la zone Rurland et celles des sols des zones avec systèmes parcellaires antiques étudiés. C. Fruchart, EPHE 2018.

La SGDBE informe sur de nombreux attributs des sols en Europe ${ }^{7}$. Nous avons pris en compte les textures, les capacités de réserve en eau et les limites à un usage agricole actuel (fig. 4). Ces paramètres donnent des informations importantes sur le potentiel agronomique des sols, en particulier sur leur aptitude à assurer l'hydratation des végétaux durant leur croissance. La texture (fig. 3 et $4 \mathrm{a}$ ) renseigne sur la granulométrie des minéraux du sol, qui conditionne son degré de rétention d'eau. Un sol argileux à la texture très fine absorbe et retient l'eau ; un sol sableux à texture grossière est perméable et peu absorbant. Quel que soit le type de zone considéré ${ }^{8}$, les sols composés de limons fins sont les plus représentés (40 à $44 \%$ ). Les sols limono-argileux sont deux fois plus fréquents dans les zones avec parcellaires $(28 \%)$ que sur la zone Rurland (14\%) et les sols limono-sableux sont rares dans les zones avec parcellaires. Les capacités de réserve en eau des sols sont données en surface (fig. 3 et 4 b) et en profondeur (fig. $4 \mathrm{~d}$ ). La réserve à plus de $50 \mathrm{~cm}$ de profondeur bénéficie aux végétaux à systèmes racinaires profonds : arbres, ligneux (vigne, notamment), certaines herbacées (luzerne, par exemple).

\footnotetext{
${ }^{7}$ La résolution du modèle est de $1 \mathrm{~km}$.

${ }^{8}$ Zone Rurland, ensemble des zones avec parcellaire, zones avec parcellaire actuellement en milieu ouvert et zones avec parcellaires actuellement sous forêt.
} 
Systèmes parcellaires et textures de sol, capacités de réserve en eau et limitations à un usage agricole

1 Basse vallée de la Seine (vers Rouen)

2 Plaine entre Tongres et Maastricht

3 Forêt de Sénart

4 Plateau briard - Melun-Sénart

5 Plateau briard - Marne-la-Vallée

6 Forêts du Sud meusien

7 Forêts des Côtes de Moselle (région de Nancy)

8 Forêt du Warndt (région de Metz)

9 Forêt de Fénétrange

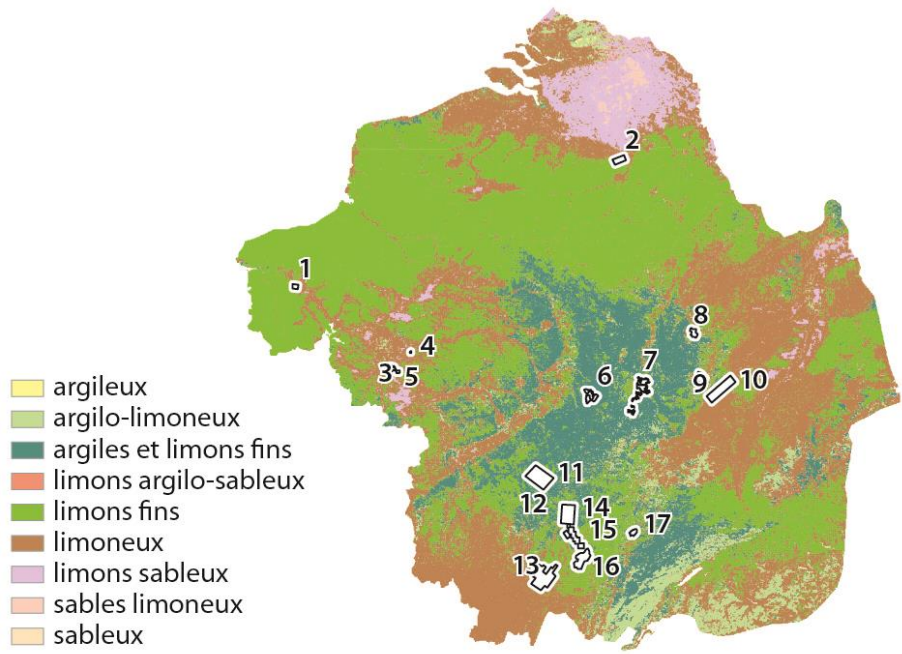

a) Textures de sol dominantes en surface
10 Piémont vosgien (entre Saverne et Saint-Quirin)

11 Massifs forestiers du Châtillonnais (vers Châtillon-sur-Seine)

12 Massif forestier entre Blessey et Salmaise (source de la Seine)

13 Systèmes parcellaires autour de Chalon-sur-Saône

14 Systèmes parcellaires autour de Mirebeau-sur-Bèze

15 Val des Tilles (région de Dijon)

16 Finage dolois

17 Forêt de Chailluz (Besançon)

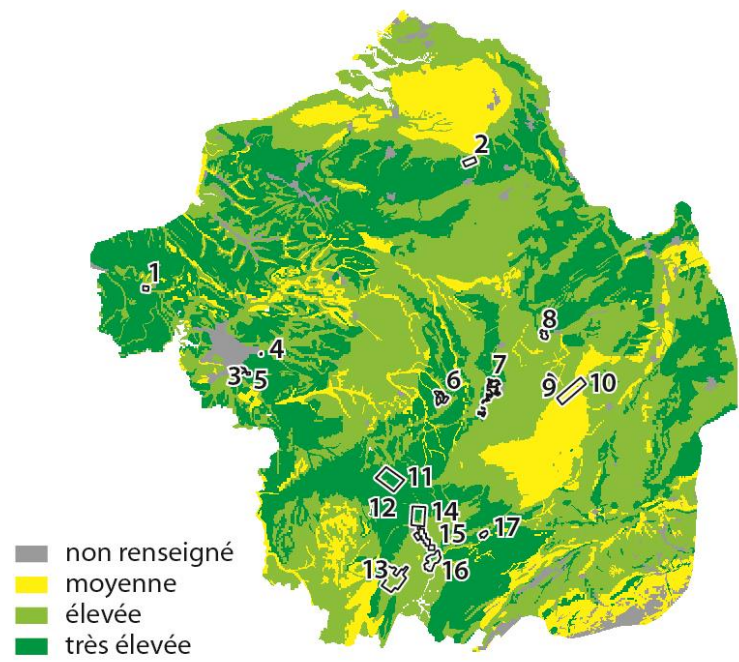

b) Capacité de réserve en eau du sol en surface

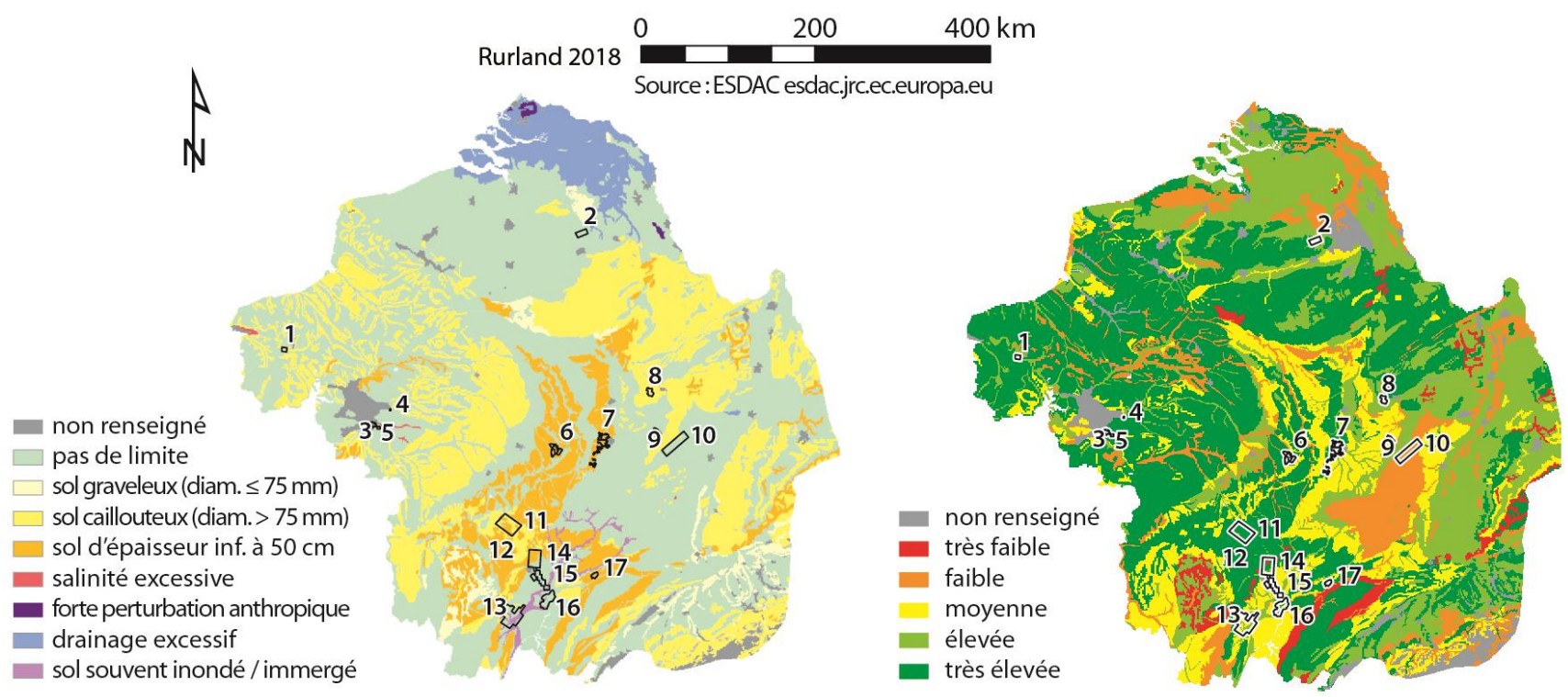

c) Principales limites à l'usage agricole actuel des sols

b) Capacité de réserve en eau à $50 \mathrm{~cm}$ et + de profondeur

Fig. 4. Cartographie des textures de sol, de la capacité de réserve en eau en surface et à partir de $50 \mathrm{~cm}$ de profondeur et des facteurs limitants dans l'aire géographique du programme Rurland. C. Fruchart, EPHE 2018 et UBFC 2019, d'après European Soil Data Centre (ESDAC), https://esdac.jrc.ec.europa.eu/, European Commission, Joint Research Centre.

Pour les 17 fenêtres d'étude, les zones aujourd'hui sous forêt présentent des différences notables par rapport aux zones en milieu ouvert : les sols avec une capacité de réserve en eau très élevée en surface et en profondeur sont fréquents sous forêt $(60 \%)$ et plus rares en milieu ouvert (29\% et $37 \%)$. Les sols avec une capacité de réserve en eau élevée en surface sont rares sous forêt $(15 \%)$ mais fréquents en milieu ouvert (68\%). Ces différences de degrés de capacité de réserve en eau entre l'état actuel forestier/non forestier suggèrent que ce facteur a peut-être influé sur la déprise ancienne d'un usage agro-pastoral dans l'Antiquité au profit d'un boisement durable, en particulier dans des zones où les capacités de réserve en eau du sol sont très élevées. 
Le modèle de limitations à l'usage agricole de la SGBDE (fig. 4c) tient compte du cadre agronomique actuel et, donc, de la mécanisation. Il présente probablement des décalages par rapport aux contraintes qui ont limité l'usage des sols durant l'Antiquité. Pour la zone Rurland, les principaux facteurs limitants actuels sont la pierrosité ( $27 \%$ de la zone) et la présence d'une couche lithique à $60 \mathrm{~cm}$ de profondeur (10\%). Parmi les fenêtres d'étude, les zones avec anciens parcellaires sous forêt contiennent plus de sols minces et/ou caillouteux (37\% et $27 \%$ ) que les zones en milieu ouvert (13\% et $4 \%$ ). $67 \%$ des zones avec anciens parcellaires en milieu ouvert ne présentent aucune limitation à un usage agricole, pour seulement $33 \%$ des zones sous forêt. Pour notre étude, nous n'avons pas tenu compte du degré d'acidité actuel des sols $(\mathrm{pH})$ et de la disponibilité en azote, phosphore et potassium (N-P-K), car les valeurs actuelles peuvent sensiblement différer des valeurs hypothétiques d'il y a deux mille ans. Un boisement prolongé tend en effet à acidifier le sol (Jabiol et al. 2011), et les niveaux de $\mathrm{N}-\mathrm{P}-\mathrm{K}$ varient avec les fertilisations et les amendements.

En résumé, l'examen à petite échelle des 17 zones avec systèmes parcellaires antiques étudiées pour le programme Rurland montre en premier lieu la diversité des contextes environnementaux dans lesquels ces systèmes se situent. Les terrains où ils ont été implantés sont plats ou en pente, les sols épais ou fins, pierreux ou non, et de textures variées. Parmi les 17 zones étudiées, ce sont celles aujourd'hui sous forêt qui offrent la plus grande diversité de contextes environnementaux. Une limitation d'ensemble est toutefois identifiable : les zones avec anciens systèmes parcellaires évitent les terrains de très faible à faible capacité de réserve en eau en profondeur.

\section{Apport du LiDAR pour l'étude de ces systèmes parcellaires}

Quel que soit le type de structure d'origine anthropique étudié, l'un des atouts majeurs du LiDAR est sa capacité à documenter la structure à grande échelle, et en même temps, à ouvrir la perspective vers une analyse à petite échelle, celle de la topographie paysagère où s'insère cette même structure. Le LiDAR nous donne simultanément accès à un point de vue synthétique et global et à une observation ponctuelle, précise et détaillée. Parallèlement, la mise en relation de la donnée topographique avec des couches d'information diverses sur Système d'Information Géographique (SIG) permet la multiplication des mises en perspective, spatiales, temporelles et disciplinaires.

Pour les systèmes parcellaires, le LiDAR nous permet, à grande échelle, d'étudier l'unité de culture parcellaire et les groupes de parcelles, et, à plus petite échelle, des systèmes entiers composés de groupes de parcelles. La petite échelle informe sur la topographie globale des systèmes parcellaires, sur la façon dont les parcelles s'agrègent en groupes, puis sur l'organisation des groupes les uns par rapport aux autres et sur leur intégration dans leur environnement. Les systèmes apparaissent souvent liés à un ou plusieurs «morphogènes ${ }^{9} »$ qui conditionnent leur organisation et leur développement. Il peut s'agir d'éléments linéaires naturels remarquables (cours d'eau, vallon) ou d'aménagements : voie ou grand fossé, par exemple. Ces tracés remarquables servent de ligne de fondation de la construction parcellaire et influencent la morphologie et l'inclinaison des parcelles. À grande échelle, l'unité parcellaire révèle des microreliefs plus ou moins ténus, témoignant des pratiques agro-pastorales passées qui ont participé à la genèse des modelés du terrain, conservés jusqu'à nos jours. Ils résultent de l'aménagement de buttes et fossés, de terrassements, de labours répétés ou d'épierrements.

\footnotetext{
${ }^{9}$ Le terme « morphogène » désigne un processus qui détermine la forme et/ou la structure d’une entité parcellaire (cf. Chouquer 2000).
} 
$n^{\circ} 2$ : Piémont vosgien (Fossé des Pandours)

$n^{\circ} 3$ : forêt domaniale de Châtillon

$\mathrm{n}^{\circ} 4$ : région de Mirebeau-sur-Bèze

$\mathrm{n}^{\circ} 5$ : Val des Tilles (Les Maillys)

$n^{\circ} 6$ : Finage dolois

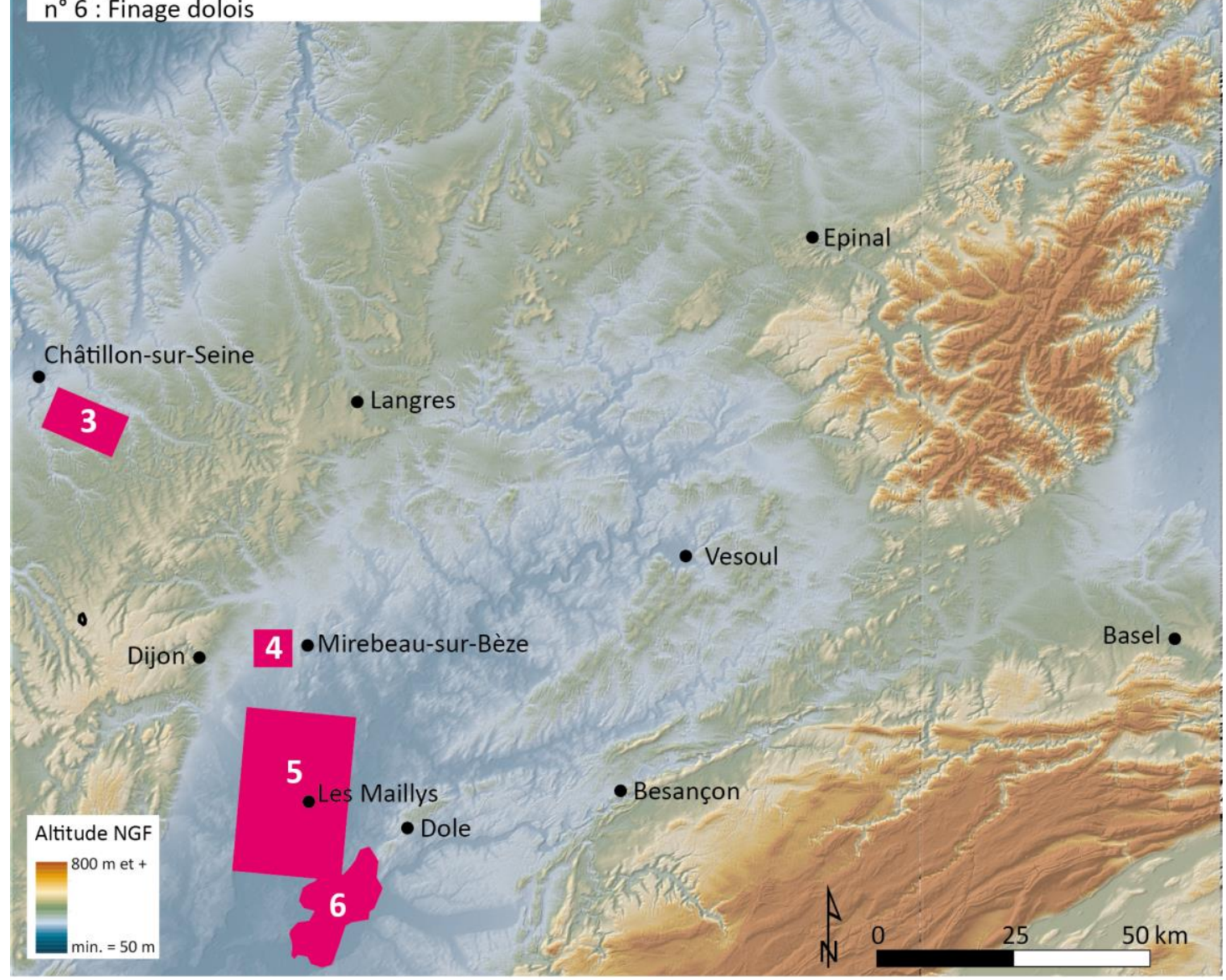

Fig. 5. Localisation des zones étudiées à l'aide de données LiDAR pour le programme Rurland. C. Fruchart, EPHE 2018 et UBFC 2019. Fond topographique d'après Copernicus Land Monitoring Service, http://www.copernicus.eu/, EU-DEM v1.0.

Pour développer l'analyse conduite dans le programme Rurland, six zones ont fait l'objet d'une étude spécifique à grande échelle, développée à partir des publications existantes et/ou de l'analyse de sources brutes, en particulier de données LiDAR (fig. 5 et tableau ci-dessous). Ces zones ${ }^{10}$ sont la forêt de Haye, située sur un plateau calcaire à l'ouest de Nancy (Meurthe-et-Moselle); le Piémont vosgien, localisé sur les collines et la basse montagne du massif gréseux des Vosges du Nord (Bas-Rhin et Moselle) ; la forêt domaniale de Châtillon, située sur un plateau calcaire au sud-est de Châtillon-surSeine (Côte-d'Or) ; le secteur des Maillys, une commune rurale de plaine occupant un territoire assez vaste au bord de la Saône, au sud-est de Dijon (Côte-d'Or); la région de Mirebeau-sur-Bèze, une autre zone en plaine de Saône au nord-est de Dijon; enfin, le Finage dolois, encore une zone de plaine, entre les rivières du Doubs et de la Saône dans le département du Jura ${ }^{11}$. Ces six zones présentent des contextes environnementaux diversifiés : territoires montagneux et de plaine, espaces actuels ouverts et forestiers, terrains avec diverses caractéristiques physico-chimiques des sols.

\footnotetext{
${ }^{10}$ Ces études pour Rurland ont été possibles grâce à la collaboration d'archéologues et chercheurs, qui ont partagé leurs données et connaissances expertes des zones : J. Bénard, G. Chouquer, J.-L. Dupouey, M. Georges-Leroy, D. Goguey, N. Meyer, A. Nüsslein et Y. Pautrat.

${ }^{11}$ Les descriptifs détaillés des recherches menées sur ces différentes zones sont librement consultables dans le volume d'annexes de la publication de synthèse du programme ERC (Reddé 2018) : https://hal.archives-

ouvertes.fr/EDITIONSAUSONIUS/public/annexes.pdf (lien valide le 1111 2019)
} 


\begin{tabular}{|c|c|c|c|c|c|c|}
\hline & Haye & Châtillon & Piémont & Maillys & Mirebeau & Finage \\
\hline $\begin{array}{l}\text { Usage du sol actuel } \\
\text { sur la zone étudiée } \\
\text { pour Rurland }\end{array}$ & Forêt & Forêt & Forêt & Agropastoral & Agropastoral & Agropastoral \\
\hline $\begin{array}{c}\text { Topographie } \\
\text { globale }\end{array}$ & $\begin{array}{c}\text { Plateau } \\
\text { calcaire sur } \\
\text { relief global de } \\
\text { type cuesta }\end{array}$ & $\begin{array}{c}\text { Plateau } \\
\text { calcaire sur } \\
\text { relief global de } \\
\text { type cuesta }\end{array}$ & $\begin{array}{l}\text { Collines et } \\
\text { montagnes } \\
\text { gréseuses }\end{array}$ & $\begin{array}{c}\text { Plaine alluviale } \\
\text { avec fort aléa } \\
\text { inondation }\end{array}$ & $\begin{array}{c}\text { Plaine alluviale } \\
\text { avec faible aléa } \\
\text { inondation }\end{array}$ & $\begin{array}{l}\text { Plaine alluviale } \\
\text { avec moyen } \\
\text { aléa inondation }\end{array}$ \\
\hline $\begin{array}{c}\text { Altitudes NGF des } \\
\text { zones étudiées }\end{array}$ & 180 à $430 \mathrm{~m}$ & 300 à 400 m & 300 à $700 \mathrm{~m}$ & 180 à $190 \mathrm{~m}$ & 190 à $250 \mathrm{~m}$ & 175 à 180 m \\
\hline $\begin{array}{c}\text { Année } \\
\text { d'acquisition du } \\
\text { levé LiDAR }\end{array}$ & 2007 & 2012 & 2007 et 2008 & ? & $?$ & $?$ \\
\hline $\begin{array}{c}\text { Surface totale du } \\
\text { relevé }\end{array}$ & $116 \mathrm{~km}^{2}$ & $380 \mathrm{~km}^{2}$ & $\begin{array}{c}10 \mathrm{~km}^{2} \\
(\mathrm{DRAC})+ \\
60 \mathrm{~km}^{2} \\
(\mathrm{RFF})\end{array}$ & $?$ & $?$ & $?$ \\
\hline $\begin{array}{c}\text { Surface étudiée } \\
\text { avec LiDAR pour } \\
\text { Rurland }\end{array}$ & $116 \mathrm{~km}^{2}$ & $110 \mathrm{~km}^{2}$ & $22 \mathrm{~km}^{2}$ & $600 \mathrm{~km}^{2}$ & $50 \mathrm{~km}^{2}$ & $200 \mathrm{~km}^{2}$ \\
\hline $\begin{array}{c}\text { Propriétaire ou } \\
\text { source de la donnée } \\
\text { LiDAR }\end{array}$ & $\begin{array}{c}\text { INRA, ONF, } \\
\text { DRAC }\end{array}$ & $\begin{array}{c}\text { Parcs } \\
\text { Nationaux de } \\
\text { France }\end{array}$ & DRAC, RFF & $\begin{array}{c}\text { IGN (RGE } \\
\text { ALTI) }\end{array}$ & $\begin{array}{l}\text { IGN (RGE } \\
\text { ALTI) }\end{array}$ & $\begin{array}{c}\text { IGN (RGE } \\
\text { ALTI) }\end{array}$ \\
\hline $\begin{array}{l}\text { Surface de zone } \\
\text { d'étude couverte } \\
\text { par LiDAR }\end{array}$ & $100 \%$ & $100 \%$ & $11 \%$ & $100 \%$ & $100 \%$ & $100 \%$ \\
\hline $\begin{array}{l}\text { Résolution du } \\
\text { MNT utilisé }\end{array}$ & $0,5 \mathrm{~m}$ & $0,5 \mathrm{~m}$ & $0,5 \mathrm{~m}$ & $1 \mathrm{~m}$ & $1 \mathrm{~m}$ & $1 \mathrm{~m}$ \\
\hline
\end{tabular}

La topographie de la forêt de Haye et celle de la forêt domaniale de Châtillon sont intégralement documentées par des données LiDAR acquises pour des recherches interdisciplinaires et notamment archéologiques. Une petite partie du Piémont vosgien, au nord de la zone d'étude sélectionnée pour Rurland, a bénéficié d'un levé LiDAR acquis pour une finalité archéologique (oppidum du Fossé des Pandours). Ce levé est complété par une acquisition LiDAR réalisée par Réseau Ferré de France (RFF) lors de l'aménagement de la ligne TGV est. Le reste du Piémont vosgien (89\% de la zone étudiée) ne disposait malheureusement d'aucun relevé LiDAR exploitable au moment de l'étude pour le programme Rurland. Ces trois zones comprenant les forêts de Haye et de Châtillon et le Piémont vosgien sont aujourd'hui presque totalement sous forêt. Les trois autres zones des Maillys, de Mirebeau-sur-Bèze et du Finage dolois sont des espaces essentiellement ouverts, à usage agropastoral. Leurs topographies détaillées sont renseignées par le Référentiel à Grande Échelle de l'IGN (RGE 
ALTI $1 \mathrm{M}^{\circledR}$ ), qui fournit un Modèle Numérique de Terrain d'une résolution de $1 \mathrm{~m}$, obtenu à partir de levés LiDAR ${ }^{12}$.

Pour notre étude, plusieurs traitements de visualisation des données altimétriques ont été réalisés à partir des MNT exploités. La figure 6 présente un aperçu de ces visualisations sur la zone d'étude du Piémont vosgien (oppidum du Fossé des Pandours). Deux ombrages simples (azimut $270^{\circ}$ et $315^{\circ}$, élévation $45^{\circ}$, fig. 6 a et b) et un modèle numérique des pentes en degrés (fig. $6 c$ ) ont été réalisés. De plus, plusieurs modèles d'index de position topographique (TPI) ont été calculés (un exemple est donné en fig. 6d). Cette dernière technique, notamment employée en géomorphologie et en écologie pour caractériser la position topographique d'éléments du relief naturel par rapport à leur contexte environnant, peut utilement être mise en œuvre en archéologie à une échelle sensiblement plus grande, celle des structures d'origine anthropique. Ceci permet de bien mettre en évidence de faibles variations altimétriques se rapportant à des entités de quelques mètres à quelques décamètres, en largeur ou longueur ${ }^{13}$.

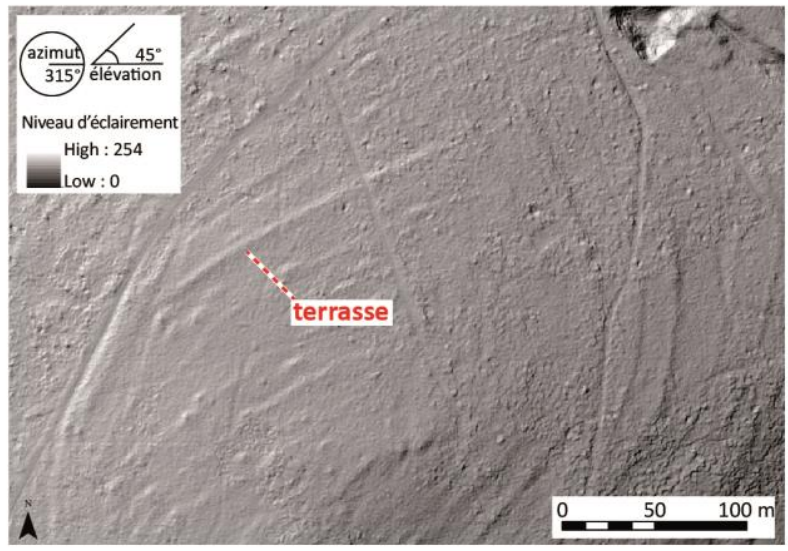

b) Visualisation par ombrage simple (outil Hillshade toolbox sur Arcgis, Spatial analyst toolbox, azimut $315^{\circ}$, élévation $45^{\circ}$ )

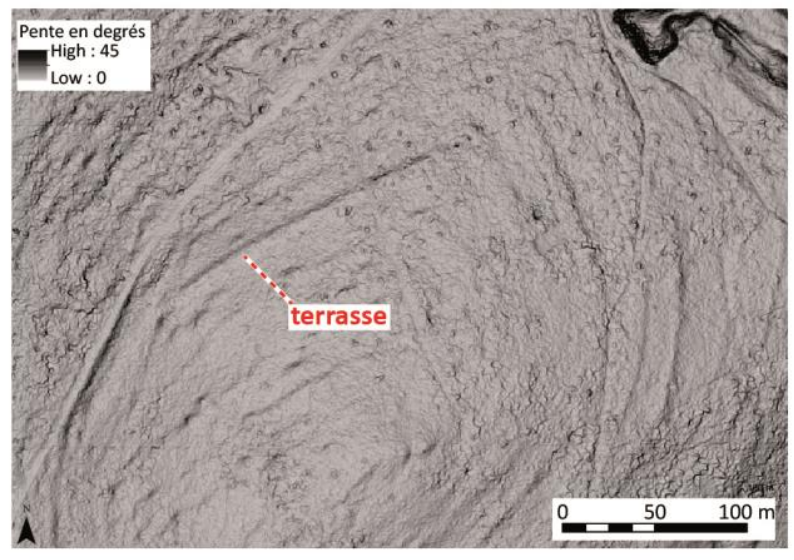

c) Visualisation par calcul des pentes en degrés (outil Slope sur Arcgis, Spatial analyst toolbox, valeurs de pente entre 0 et $45^{\circ}$ ).

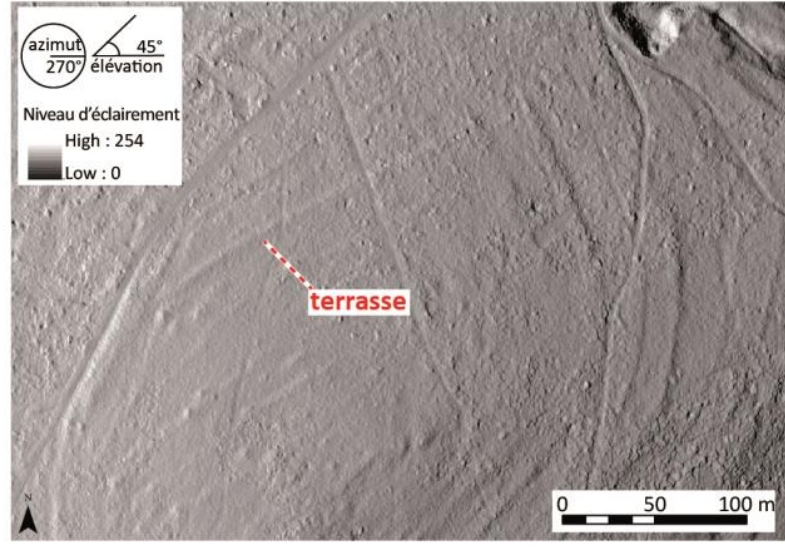

b) Visualisation par ombrage simple (outil Hillshade toolbox sur Arcgis, Spatial analyst toolbox, azimut $270^{\circ}$, élévation $45^{\circ}$ )

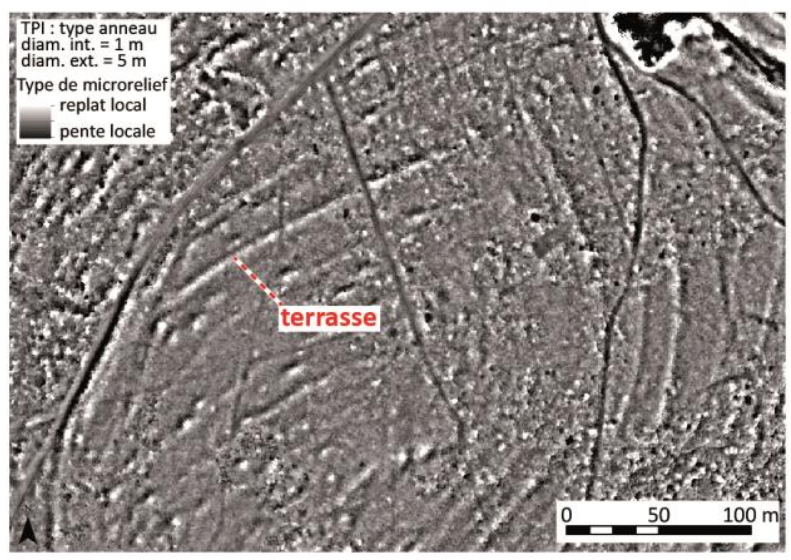

d) Visualisation par index de position topographique (outil TPI Jeness toolbox sur Arcgis, mode anneau, diamètre intérieur $1 \mathrm{~m}$, diamètre extérieur $5 \mathrm{~m}$.

Fig. 6. Différentes visualisations de systèmes en terrasse sur l'oppidum du Fossé des Pandours. C. Fruchart, EPHE 2016 et UBFC 2019, d'après données LiDAR LGV Est, RFF 2008 et Fossé des Pandours, CD 67Archéologie Alsace.

\footnotetext{
${ }^{12}$ Un descriptif des caractéristiques des modèles numériques 3D du RGE ALTI ${ }^{\circledR}$ Version 2.0 est consultable sur le site de I'IGN : http://professionnels.ign.fr/doc/DC RGEALTI 2-0.pdf (lien valide le 1111 2019).

${ }^{13}$ Pour plus d'information sur cet outil de visualisation, on peut se reporter au poster consultable à l'adresse suivante (lien valide le 1111 2019) : http://www.jennessent.com/arcview/TPI jen poster.htm. Par ailleurs, un ouvrage présentant de façon détaillée une grande variété de traitements de visualisation de données LiDAR (Kokalj, Hesse 2017) est librement consultable en ligne : https://iaps.zrc-sazu.si/sl/publikacije/airborne-laser-scanning-raster-data-visualization (lien valide le 11112019 ).
} 
L'apport du LiDAR a été multiple pour notre étude de systèmes parcellaires antiques. Tout d'abord, le recours au LiDAR a permis d'étudier des zones situées aussi bien en forêt qu'en milieu ouvert. Nous observons ainsi l'extension significative d'aménagements anciens à vocation agropastorale dans des forêts actuelles, ce qui pose la question des changements historiques de l'attractivité des sols pour leur usage agropastoral. L'abandon ancien de l'usage agropastoral a-t-il été principalement motivé par des facteurs environnementaux (sols trop peu fertiles ou trop secs, par exemple) ou par des déterminants sociétaux (éloignement des zones de peuplement, problèmes d'accessibilité, par exemple) ? Nos travaux pour le programme Rurland ont aussi montré que les vestiges de systèmes parcellaires anciens sont encore détectables dans des zones ouvertes et mises en culture actuellement. L'attribution (totale ou partielle) à l'Antiquité des systèmes parcellaires mis en évidence dans ces zones ouvertes a été obtenue en croisant les relevés topographiques établis à partir de données LiDAR avec des informations archéologiques issues de bases de données se rapportant à des fouilles et des prospections, et en complément avec d'autres informations, telles que des archives historiques. En faisant appel à un processus hypothético-déductif, nous avons pu associer des entités archéologiques datées et précisément localisées avec les vestiges de structures à vocation agropastorale identifiées grâce aux micro-variations du relief qu'elles occasionnent.

Notre étude a aussi montré qu'on peut étudier des systèmes parcellaires anciens à partir du RGE $\mathrm{ALTI}^{\circledR}$, malgré sa résolution limitée à $1 \mathrm{~m}$, à condition que les MNT des zones étudiées aient été établis à partir de levés LiDAR. Bien sûr, la restitution de la microtopographie est moins fine et détaillée que celle obtenue à partir de relevés pour lesquels les MNT offrent une résolution de $0,5 \mathrm{~m}$. Mais l'échelle nécessaire pour l'analyse des structures composant des systèmes parcellaires est compatible avec la résolution du RGE $\mathrm{ALTI}^{\circledR}$, car les limites de parcelles, les tronçons de voies, les aménagements en terrasses, etc., qui composent ces systèmes sont formés de linéaments longs de plusieurs dizaines de mètres et larges de plusieurs mètres, ce qui est parfaitement détectable avec une résolution de $1 \mathrm{~m}^{14}$. En revanche, la précision obtenue à partir d'une altimétrie construite par photogrammétrie, même en milieu ouvert, s'avère insuffisante, car les microreliefs restitués sont trop peu détaillés pour permettre une exploitation à finalité archéologique.

\footnotetext{
${ }^{14}$ Les figures suivantes présentent plusieurs profils d'aménagements parcellaires et de tronçons viaires qui permettent d'apprécier les largeurs de ces structures.
} 


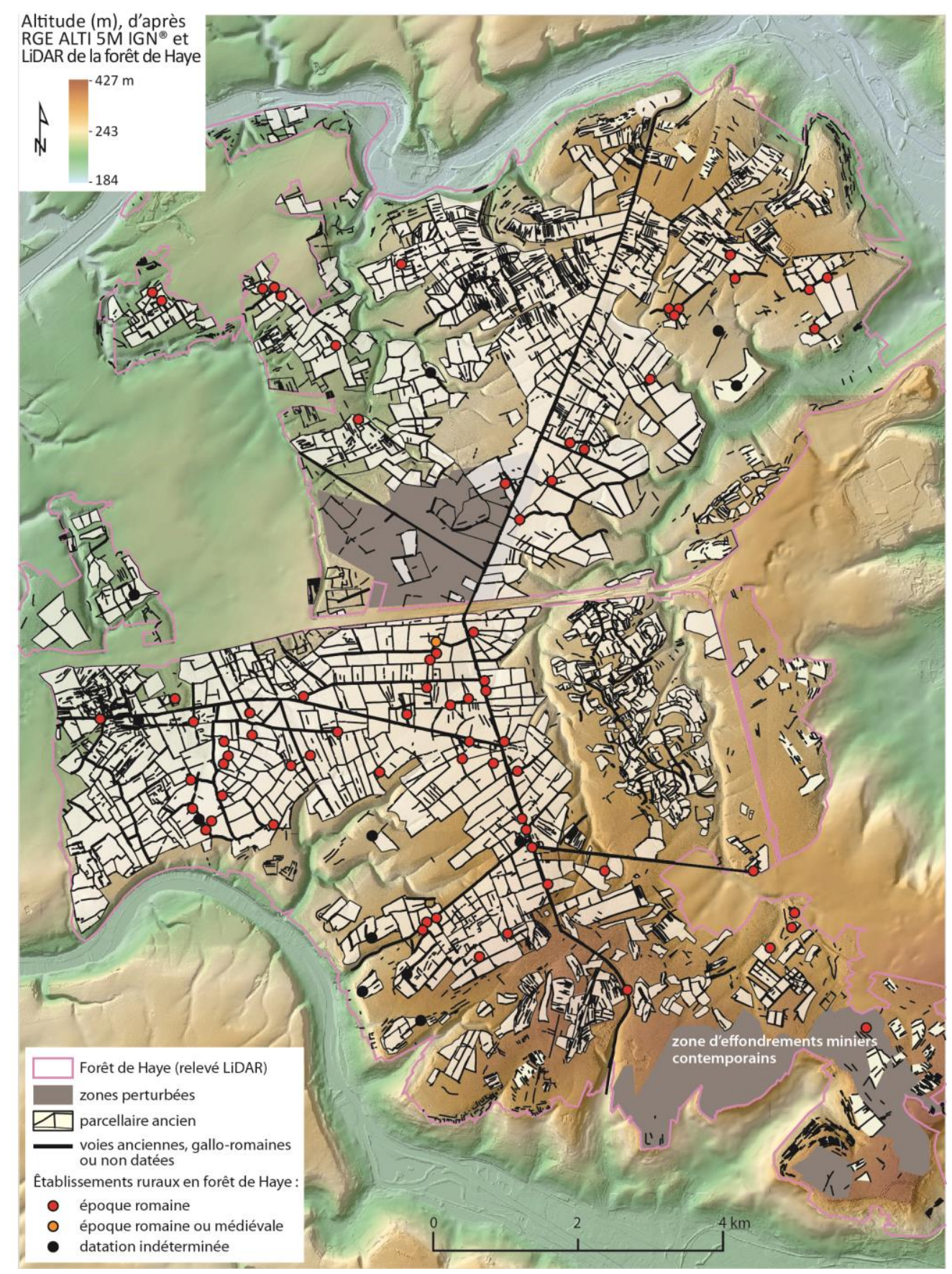

Fig. 7. Forêt de Haye. Proposition de regroupement de parcelles par blocs en superposition avec la voirie et les établissements. C. Fruchart, EPHE 2017 et UBFC 2019, d'après données archéologiques M. GeorgesLeroy, DRAC Grand-Est et données LiDAR Haye 2007, DRAC Lorraine, INRA Nancy, ONF et RGE ALTI 5M $I G N^{\circledR}$.

Un autre apport du LiDAR a été de révéler la variété des morphologies agraires anciennes qui sont encore détectables aujourd'hui. Les figures 7 à 10 montrent quelques exemples des formes d'aménagements agro-pastoraux que nous avons pu identifier : des systèmes parcellaires composés de parcelles polygonales de formes et de tailles diverses, agglomérées en groupes plus ou moins étendus. Ce type d'aménagements (fig. 7) compose l'essentiel des systèmes parcellaires étudiés en forêt de Haye, en forêt domaniale de Châtillon et dans la plaine à l'ouest de Mirebeau-sur-Bèze. Les systèmes parcellaires identifiés dans la partie nord du Piémont vosgien (oppidum du Fossé des Pandours et ses alentours, fig. 8) sont essentiellement formés de structures en terrasses aménagées sur des sols 
relativement pentus ${ }^{15}$. Sur le Finage dolois (fig. 9), les données du RGE ALTI ont permis de mettre en évidence des séries de champs surélevés, type d'aménagements agraires sur sols humides bien connu en Amérique du Sud, aussi bien à des périodes archéologiques remontant à 2500 ans qu'encore en exploitation aujourd'hui (Mollard, Walter 2008). Enfin, dans le secteur des Maillys (fig. 10), l'exploitation du RGE ALTI $^{\circledR}$ a permis d'identifier des systèmes parcellaires connus en Europe du Nord sous le nom de «Celtic» fields, depuis les périodes protohistoriques jusqu'à l'époque moderne (Allemagne, Pays-Bas : Arnoldussen, Van der Linden. 2017).

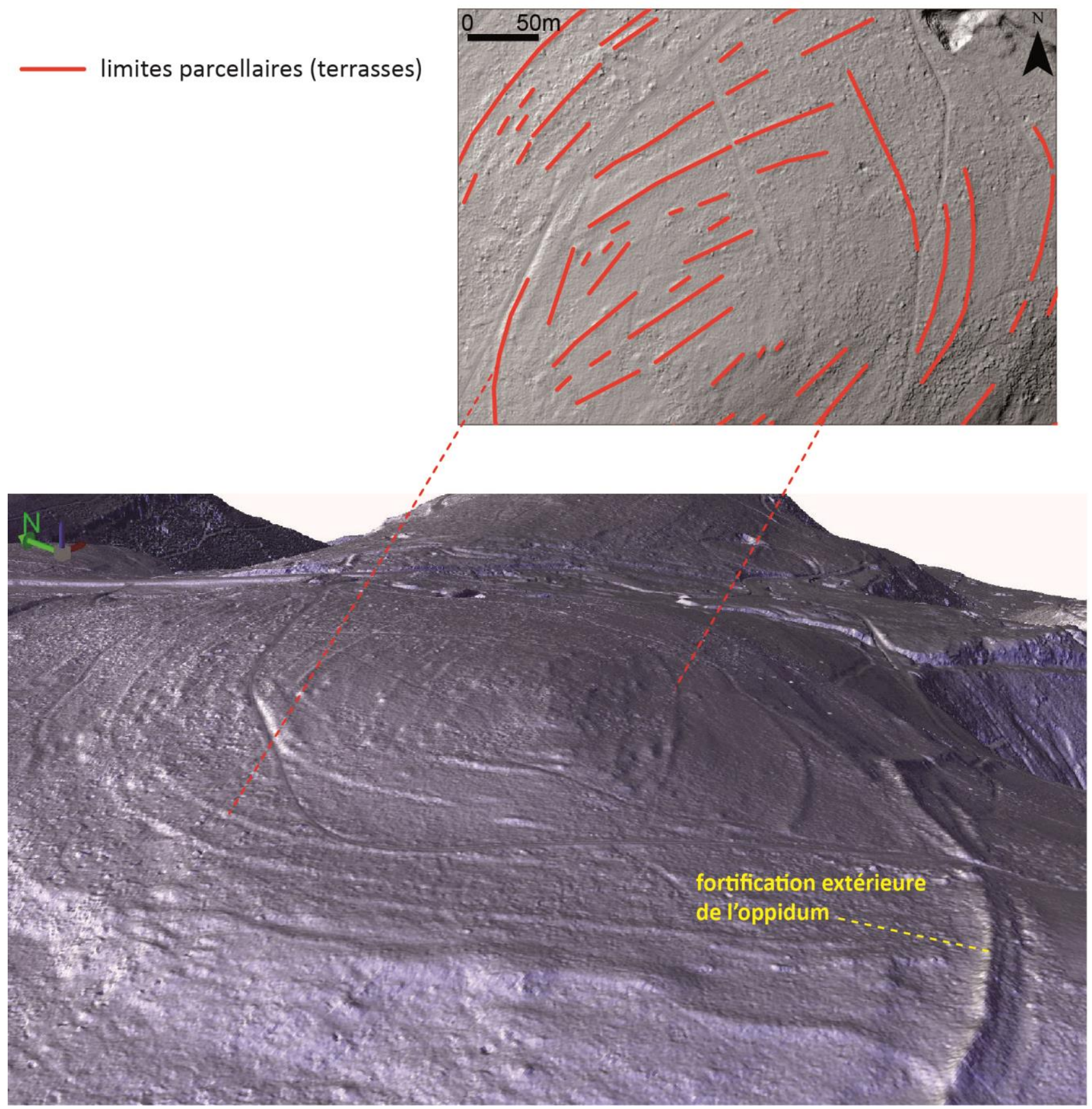

Fig. 8. Zone du Piémont vosgien, oppidum du Fossé des Pandours. Aperçu en vue volumétrique et en vue en plan d'aménagements interprétés comme des terrasses à vocation agro-pastorale. C. Fruchart, EPHE 2016 et UBFC 2019, d'après données LiDAR LGV Est, RFF 2008 et Fossé des Pandours, CD 67-Archéologie Alsace.

\footnotetext{
${ }^{15}$ Les prospections menées systématiquement depuis les années 1990 sur l'ensemble du Piémont vosgien par des archéologues experts en techniques de prospection au sol sous forêt, ainsi que les résultats de plusieurs fouilles, indiquent la présence récurrente de systèmes parcellaires formés de terrasses matérialisées par des murs de soutènement en pierres sèches. La datation romaine est établie par le mobilier découvert en prospection et en fouille.
} 

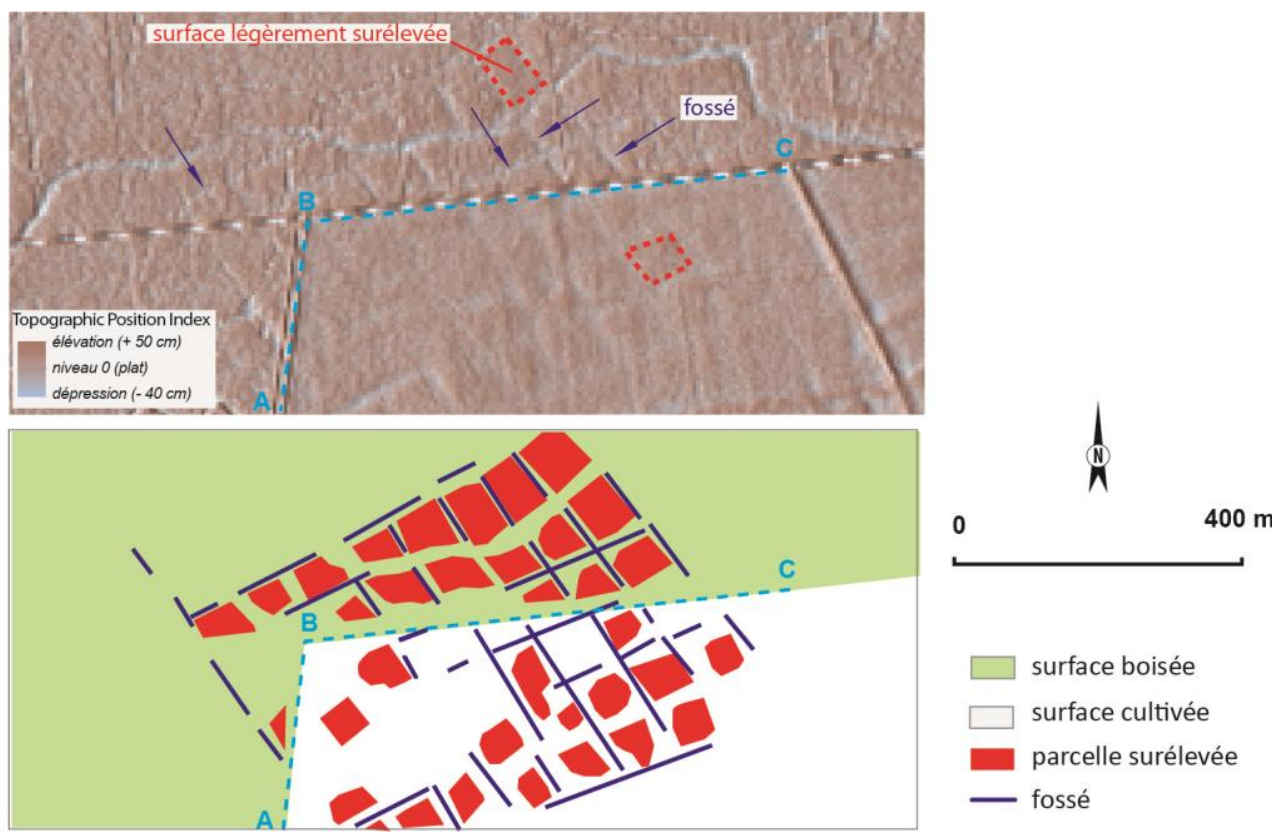

a) Anciens champs surélevés détectés sur la zone d'étude du Finage dolois, au lieu-dit Les Breuvées, àTavaux

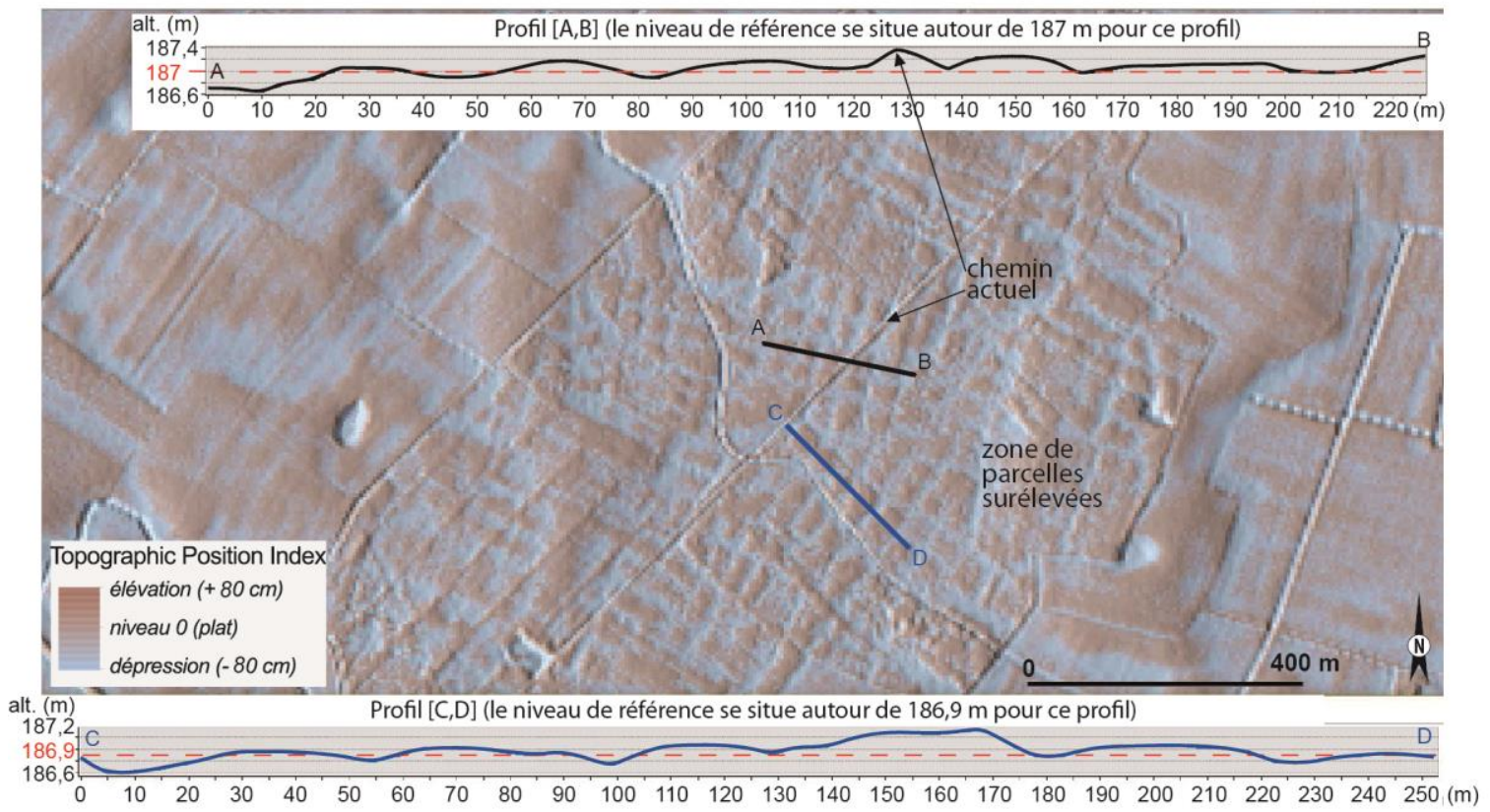

b) Anciens champs surélevés détectés sur la zone d'étude du Finage dolois, dans le Bois des Noues à Aumur.

Fig. 9. Finage dolois. Aperçus de groupes de champs surélevés, sous forêt et en zone ouverte aujourd'hui, obtenus à partir de données LiDAR du RGE ALTI 1M de I'IGN. C. Fruchart, EPHE 2017 et UBFC 2019, d'après RGE ALTI IGN ${ }^{\circledR}$. 

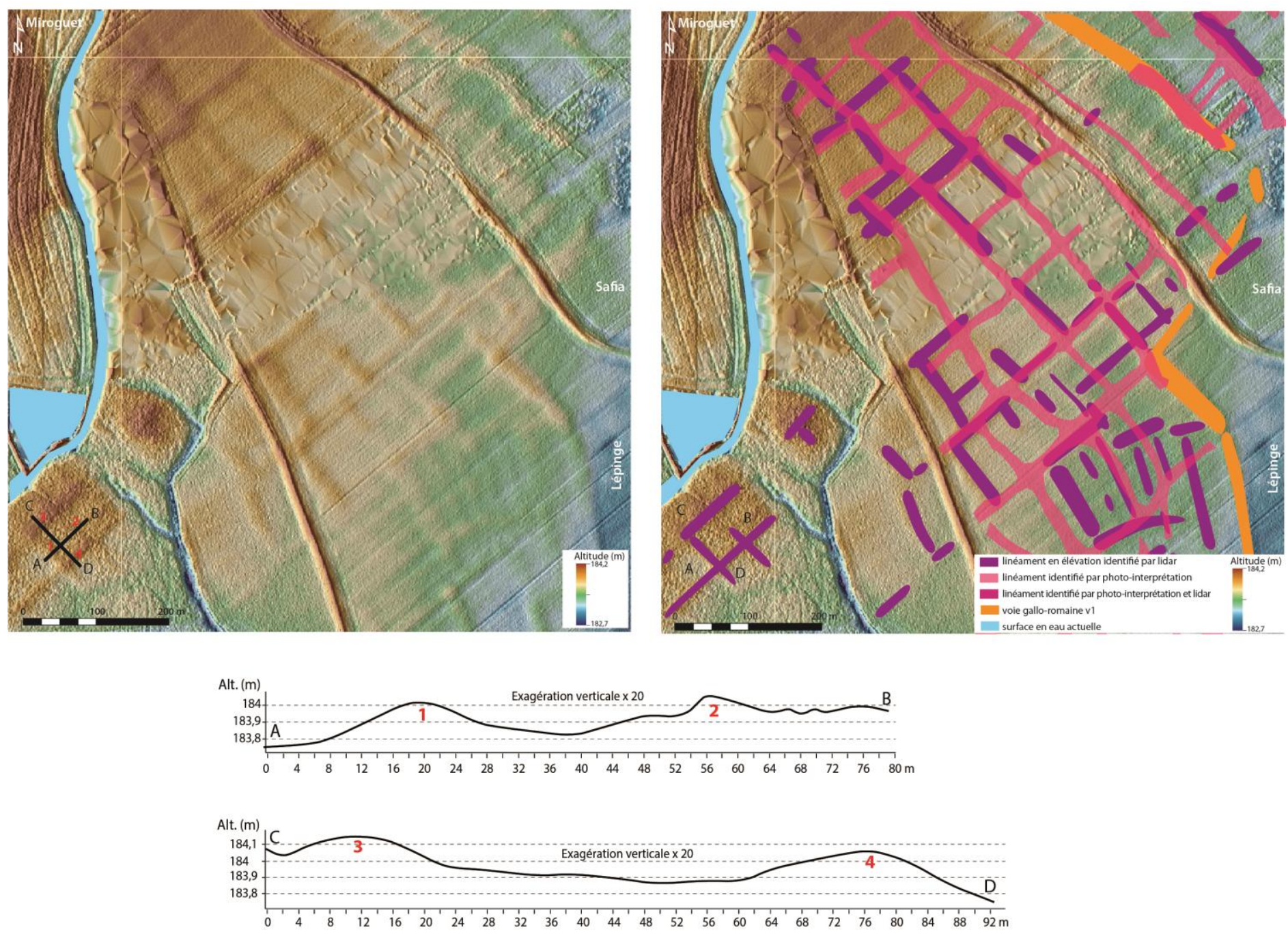

Fig. 10. Les Maillys. Aménagements parcellaires formant des structures alvéolées de type "Celtic » fields. $C$. Fruchart, EPHE 2017 et UBFC 2019, d'après RGE ALTI IGN ${ }^{\circledR}$.

\section{Conclusion}

En somme, le LIDAR renseigne la topographie et l'aménagement de notre territoire avec une précision, une extension et une continuité spatiale encore jamais atteintes en archéologie auparavant. Ceci nous permet de mener des travaux d'analyse spatiale inscrits dans des contextes précis, locaux, tout en procédant à des mises en perspective couvrant des territoires étendus, jusqu'à plusieurs centaines de kilomètres carrés d'un seul tenant. Nous ignorons évidemment le degré d'exhaustivité du relevé des structures anciennes obtenu grâce à cet outil de télédétection, mais d'une manière générale en archéologie, nous savons que l'information avec laquelle nous sommes contraints de travailler est toujours, ou presque, lacunaire.

Les recherches sur les paysages anciens mobilisent diverses disciplines : archéologie, histoire, géographie, géomatique, sciences de l'environnement. Elles aident à produire des hypothèses et modèles pour mieux comprendre les cadres de vie passés et leurs évolutions, en exploitant des sources variées : fouilles, prospections, photographies aériennes et archives, entre autres. Le LiDAR, qui est intégré à ces recherches depuis une quinzaine d'années, fait considérablement progresser nos connaissances en archéologie des espaces forestiers, à l'instar des progrès obtenus sur sols nus ou cultivés avec la photographie aérienne. L'information exploitable, jusque-là très fragmentée, documente à présent de vastes espaces de façon quasi continue. Couplé aux sources de données traditionnelles, aux SIG et à la modélisation, le LiDAR ouvre de nouvelles perspectives pour mieux connaître les paysages passés et leurs transformations. 
L'examen à grande échelle des systèmes parcellaires anciens permet d'étudier les morphologies parcellaires et la structuration des parcelles les unes par rapport aux autres. Un examen à grande et petite échelle prenant en compte des caractéristiques environnementales - topographie, caractéristiques physiques et chimiques du sol - permet d'identifier quelques facteurs qui semblent favorables ou défavorables à un usage agro-pastoral passé de territoires. Une capacité de réserve en eau du sol insuffisante pourrait, par exemple, être un facteur limitant cet usage dans l'Antiquité. Pour pouvoir faire cette observation, le LiDAR est un outil précieux car il nous permet de dépasser le contexte actuel d'usage des sols. Nous pouvons examiner indifféremment des milieux actuels cultivés, en prairies ou forestiers, et donc, intégrer de facto une dimension dynamique à notre approche du paysage, en dissociant l'usage actuel du sol de celui qu'il a eu au cours des siècles passés. Ceci nous amène à poser la question des facteurs d'attractivité des sols dans l'Antiquité, peut-être très différents des facteurs actuels $^{16}$. La diversité physico-chimique naturelle des sols, la variété de leurs expositions et des contextes climatiques auxquels ils sont soumis permettent des productions agricoles diversifiées que des savoir-faire ont pu mettre en valeur autrefois, sans mécanisation ni connaissances agronomiques contemporaines. De tels savoir-faire existaient encore en France au siècle dernier, et sont toujours vivants en divers endroits du monde peu industrialisés. Les futures études archéologiques de systèmes parcellaires révélés par le LiDAR gagneront probablement à intégrer ces facteurs géopédologiques dans une mise en perspective dynamique du paysage agro-pastoral.

\section{Références}

Abadie, J., J.-L. Dupouey, C. Avon, X. Rochel, A. Salvaudon, T. Tatoni \& L. Bergès 2017. «Déterminants du changement du couvert forestier depuis 1860 dans le parc naturel régional du Luberon et implications pour la répartition des forêts actuelles », Revue Forestière Française, LXIX, 4-5 : 371-386

Arnoldussen, S. \& M. Van der Linden 2017. "Palaeo-ecological and archaeological analysis of two Dutch Celtic fields (Zeijen-Noordse Veld and Wekerom-Lunteren): solving the puzzle of local Celtic field bank formation", Vegetation History and Archaeobotany, 26, $6: 551-570$.

Bispo, A., C. Guellier, E. Martin, J. Sapijanskas, H. Soubelet, \& C. Chenu 2016. Les sols : intégrer leur multifonctionnalité pour une gestion durable. Versailles, Éditions Quae, p.

Bourrigaud, R. \& F. Sigaut (dir.) 2007. Nous labourons. Actes du colloque "Techniques de travail de la terre, hier et aujourd'hui, ici et là-bas ». Nantes, Centre d'Histoire du Travail, 399 p.

Chase, D. \& A. Chase 2017. "Caracol, Belize, and Changing Perceptions of Ancient Maya Society", Journal of Archaeological Research, 25 : 185-249.

Chouquer, G. 2000. L'étude des paysages. Essais sur leurs formes et leur histoire. Paris, Errance, 208 p.

Crow, P., S. Benham, B. J. Devereux \& G. S. Amable 2007. "Woodland vegetation and its implication for archaeological survey using LiDAR", Forestry, 80, $3: 241-252$.

Crozet, A., C. Laplaige \& X. Rodier 2018. «Une approche multidisciplinaire de la fabrique des paysages dans la longue durée dans les forêts de Blois, Russy, Boulogne et Chambord (Loir-et-Cher)», Projets de paysage : revue scientifique sur la conception et l'aménagement de l'espace. École Nationale Supérieure du Paysage de Versailles, [en ligne], mis en ligne le 06 juillet 2017, consulté le 31 juillet 2019 . URL: https://www.projetsdepaysage.fr/editpdf.php?texte=949.

Crutchley, S. \& H. Winton 2010. "Using lidar in conjunction with other investigation techniques in Savernake Forest, Marlborough. Past landscapes beneath the trees. Using Lidar in Woodlands", Proceedings of a one-day conference at The Park Campus. University of Gloucestershire : 35-56.

Étienne, D. 2011. Les mardelles intra-forestières de Lorraine. Origines, archives paléoenvironnementales, évolutions dynamiques et gestion conservatoire, Thèse de doctorat en Géosciences. Nancy, université de Nancy 1.

\footnotetext{
${ }^{16}$ Voir Gras 1994 ; Mazoyer et Roudard 1997 ; Bourrigaud et Sigaut 2007 ; Mollard, Walter 2008 ; Ruellan 2010, Bispo et al. 2016, notamment.
} 
Favory, F., N. Bernigaud, C. Fruchart \& P. Nowicki 2017. "Chapitre 17. Le Finage dolois", in Reddé M. (dir.). Gallia Rustica 1. Les campagnes du nord-est de la Gaule de la fin de l'âge du Fer à l'Antiquité tardive. Bordeaux, Éditions Ausonius, Collection Mémoires, 49 : 817-867.

Favory, F. \& C. Fruchart 2018. "Chapitre 7. Les systèmes parcellaires tardo-laténiens et gallo-romains" in Reddé M. (dir.). Gallia Rustica. 2, Les campagnes du Nord-Est de la Gaule, de la fin de l'âge du Fer à l'Antiquité tardive. Bordeaux, Éditions Ausonius, Collection Mémoires, 50 : 401-451.

Forlin, P. 2012. "Airborne LiDAR data analysis within the Alpine landscapes of Trentino: a methodological approach", Post Classical Archaeologies, 2 : 247-268.

Froehlicher, L. 2016. Les haies, une alternative à l'openfield dans les zones loessiques d'Alsace? Perspectives historiques, systèmes agraires du futur, érosion, effets sur le colluvionnement et le stockage du carbone, Thèse de doctorat en Géographie. Strasbourg, Université de Strasbourg.

Fruchart, C. 2014. Analyse spatiale et temporelle des paysages de la forêt de Chailluz (Besançon, Doubs) de l'antiquité à nos jours, Thèse de doctorat en Archéologie. Besançon, Université de Franche-Comté.

Georges-Leroy, M., J. Bock, É. Dambrine \& J.-L. Dupouey 2009. «Le massif forestier, objet pertinent pour la recherche archéologique. L'exemple du massif forestier de Haye (Meurthe-et-Moselle) », Revue Géographique de l'Est, 49, 2 3, [en ligne], mis en ligne le 01 avril 2013, consulté le 31 juillet 2019 . URL: http://journals.openedition.org/rge/1931.

Goguey, D. \& J. Bénard (dir.) 2018. Structures en pierre du plateau du Châtillonnais (Côte-d'Or). Du Hallstatt à l'Antiquité tardive. L'apport de l'archéologie forestière. Drémil-Lafage, Éditions Mergoil, Collection Archéologie et histoire romaine, $39,289 \mathrm{p}$.

Gras, R. 1994. Sols caillouteux et production végétale. Paris, Édition Quae, 175 p.

Heinzel, J. \& B. Sittler 2010. "LiDAR surveys of ancient landscapes in SW Germany: Assessment of archaeological features under forests and attempts for automatic pattern recognition", British Archaeological Reports. International Series, 2118 : 113-122.

Jabiol, B., G. Lévy, M. Bonneau \& A. Brêthes 2011 Comprendre les sols pour mieux gérer les forêts : contraintes et fragilités des sols, choix des essences, précautions sylvicoles, améliorations. Nancy, AgroParisTech - Engref, 624 p.

Johnson, K. M. \& W. B. Ouimet 2016. "Physical properties and spatial controls of stone walls in the northeastern USA: Implications for Anthropocene studies of $17^{\text {th }}$ to early $20^{\text {th }}$ century agriculture", Anthropocene, $15: 22-36$.

Kokalj, Z., Hesse R. 2017. Airborne laser scanning raster data visualization. Založba ZRC SAZU 14

Laplaige, C. 2012. Comparaison de signaux (géophysique, LiDAR) utilisés dans l'étude des dynamiques anthropiques et naturelles, Thèse de doctorat en Archéologie. Besançon, Université de Franche-Comté.

Laüt, L. 2018. «L'exploitation archéologique des données LiDAR sur la forêt de Tronçais : un premier bilan », Bulletin de la société des amis de la forêt de Tronçais, 63 : 43-59.

Le Jeune, Y., C. Dardignac \& S. David 2017. «Bercé avant la forêt. Premiers résultats de l'exploitation des cartes anciennes et des données lidar sur la forêt de Bercé (Sarthe)», Revue Forestière Française, 69 : 519-543.

Mazoyer, M. \& L. Roudart 1997. Histoire des agricultures du monde. Du néolithique à la crise contemporaine. Paris, Seuil, 534 p.

Mc Coy, M. D., G. P. Asner \& M. W. Graves 2011. "Airborne lidar survey of irrigated agricultural landscapes: an application of the slope contrast method", Journal of Archaeological Science, 38, 9 : 2141-2154.

Mollard, É. \& A. Walter 2008. Agricultures singulières. Paris, IRD Éditions, 343 p.

Opitz, R., K. Ryzewski, J. Cherry \& B. Moloney 2015. "Using Airborne LiDAR Survey to explore Historic-era archaeological landscapes of Montserrat in the Eastern Caribbean", Journal of Field Archaeology, 41, 1 : 523-541.

Reddé, M. (dir.) 2017. Gallia Rustica 1. Les campagnes du nord-est de la Gaule de la fin de l'âge du Fer à l'Antiquité tardive. Bordeaux, Éditions Ausonius, Collection Mémoires, 49, 868 p.

Reddé, M. (dir.) 2018. Gallia Rustica 2. Les campagnes du nord-est de la Gaule, de la fin de l'âge du Fer à l'Antiquité tardive. Bordeaux, Éditions Ausonius, Collection Mémoires, 50, 717 p.

Ruellan, A. 2010. Des sols et des hommes : un lien menacé, Bondy, IRD Éditions, 108 p.

Stular, B. 2011. "The use of lidar-derived relief models in archaeological topography. The Kobarid region (Slovenia) case study", Arheološki vestnik, $62: 393-432$. 